\title{
Coexistence of Aedes aegypti and Aedes albopictus in Jinghong City, Yunnan Province: A Survey of Aedes aegypti Invasion
}

\section{Yu-Hong Guo ${ }^{a \#}$, Sheng-Jie Lai ${ }^{\mathrm{b}, \mathrm{c}, \mathrm{d \#}}$, Qiang Huang ${ }^{\mathrm{e}}$, Dong-Sheng Ren ${ }^{\mathrm{a}}$, Jian-Hong Zou ${ }^{\mathrm{e}}$, Qi-Yong Liu ${ }^{\mathrm{a}}$, Huai-qing Zhang ${ }^{\mathrm{f}}$}

a WHO Collaborating Centre for Vector Surveillance and Management, State Key Laboratory of Infectious Disease Prevention and Control, Collaborative Innovation Center for Diagnosis and Treatment of Infectious Diseases, National Institute for Communicable Disease Control and Prevention, Key Laboratory of Surveillance and Early-warning on Infectious Disease, Chinese Center for Disease Control and Prevention, Beijing, 102206, China

${ }^{b}$ Division of Infectious Disease, Key Laboratory of Surveillance and Early-warning on Infectious Disease, Chinese Center for Disease Control and Prevention, Beijing 102206, China

c WorldPop, Department of Geography and Environment, University of Southampton, Southampton SO17 1BJ, UK

a School of Public Health, Fudan University, Key Laboratory of Public Health Safety, Ministry of Education, Shanghai, China

e Jinghong Center for Disease Control and Prevention, Jinghong City 666100, Xishuangbanna, Yunnan province, China

${ }^{f}$ The People's Bank of China, Beijing 100800, China

*Corresponding author: Qi-Yong Liu, Department of Vector Biology and Control, National Institute for Communicable Disease Control and Prevention, PI for Disease Vectors, State Key Laboratory of Infectious Disease Prevention and Control, China CDC, and Key Laboratory of Surveillance and Early-Warning on Infectious Disease, Chinese Center for Disease Control and Prevention, 155, Changbai Road, Changping, Beijing-102206, China, Fax: +86 1058900739. E-mail: liuqiyong@icdc.cn

\# These authors have contributed equally.

Received date: December 04, 2016; Accepted date: December 16, 2016; Published date: December 21, 2016

Copyright:@ 2016 Guo YH, et al. This is an open-access article distributed under the terms of the Creative Commons Attribution License, which permits unrestricted use, distribution, and reproduction in any medium, provided the original author and source are credited.

\begin{abstract}
We conducted this study to confirm that Aedes aegypti has invaded and colonized in Xishuangbanna, Yunan province, China. And we conducted a larval survey for dengue mosquito vectors, Ae. aegypti and Ae. albopictus, to assess on possible competition for breeding sites between these both species in urban environment in Jinghong city. The study was carried out at least once a month in February and March, November and December, and three times for each month from April to October in 2014 in five communities in the municipality of Jinghong city. No less than 50 houses were surveyed each time in one place. We collected immature stages of mosquitoes from all types of breeding sites in houses and their premises. Morphological species identification was carried for Ae. aegypti and Ae. albopictus. Both species occurred in all surveyed areas. Aedes aegypti has occupied a considerable composition $(67.75 \%)$ since the uncertain time of its first invasion to Jinghong city. Aedes aegypti was predominant species indoor $(74.19 \%)$, but also presented in surrounding environment $(25.81 \%)$; while Ae. albopictus favored the outdoor environment $(54.55 \%)$ as well as in houses $(45.45 \%)$. We gave the preference water containers (tires) for dengue vectors, described different distribution in different investigated places, and showed with monthly variance by indices of $\mathrm{BI}$ and $\mathrm{Cl}$. We gave our rational explanation for the variance with raining in this area; Aedes keep a high density value during the rainy season. For both dengue vectors, the possibility cannot be excluded that the invasion of $A e$. aegypti in the city might lead to a decrease or elimination of Ae. albopictus in houses and dwellings. Furthermore, we also found that both dengue vectors have already occupied the breeding sites in houses and their premises with water container in Jinghong city, and both should be managed during dengue epidemic season. These findings from this study are relevant for dengue vector control programs.
\end{abstract}

Keywords: Breeding; Morphological; Species; Identification; Ecological; Vector

\section{Introduction}

Dengue outbreaks have occurred frequently in Xishuangbanna, Yunnan province during recent years, since the first serious dengue outbreak in 2013 [1]. According to previous studies [2,3], the distribution of Aedes aegypti in China is only limited in some areas in Guangdong, Guangxi and Hainan provinces. For the first time, it was [4] elaborated that the distribution of Ae aegypti in Dehong DaiJingpo Autonomous Prefecture, Yunnan province, China. According to previous studies [1], it was reported that Ae. aegypti was detected for the first time in Xishuangbanna, and we found the two important dengue vectors, Ae. Aegypti and Ae. Albopictus co-exist in Jinghong city, while we carried out the vector management during the dengue outbreak in 2013. Although it was [5] revealed that dengue vectors ( $A e$ aegypti and Ae. albopictus), given the impressive dispersal abilities, are likely to spread into actual distributional shifts at eastern China, but no occurrence data were available included eastern China. Also, it was [6] described that the global distribution of Ae. aegypti and Ae. albopictus without distribution of Ae. aegypti in China. Thus, there are no records for the distribution of Ae. aegypti in Jinghong City until the current study. We conceived our study in 2013, with the intention to determine the dengue vector species composition, and the breeding habitat preference in these areas. We hypothesized that most vectors reproduced in all types of container breeding sites in houses and peridomestic areas, this consideration prompted us to explore our surveillance on houses and it's vicinities in Jinghong city. The result will be used for vector control programs which will focus on elimination or treating the key breeding sites for dengue vectors to reduce their density below a target threshold and prevent dengue outbreaks. The results will guide us for targeted treatment. 
Citation: Guo YH, Lai SH, Huange Q, Rena DS, et al. (2016) Coexistence of Aedes aegypti and Aedes albopictus in Jinghong City, Yunnan Province: A Survey of Aedes aegypti Invasion. J Trop Dis 4: 227. doi:10.4172/2329-891X.1000227

Page 2 of 6

\section{Materials and Methods}

Study sites: Our study was conducted in five localities, which were the east (Gaozhuang), south (Manlongkuang), west (Damanme), north (Tiancheng), and center (Nakunkang) of Jinghong city (Figure 1). The five localities possess typical ecological characteristics of Jinghong city and locate at different regions with relatively abundant water containers, concentrated population distribution, good traffic conditions for commercial trade. The five localities were randomly selected with the intention to represent the overall situation of Jinghong City.

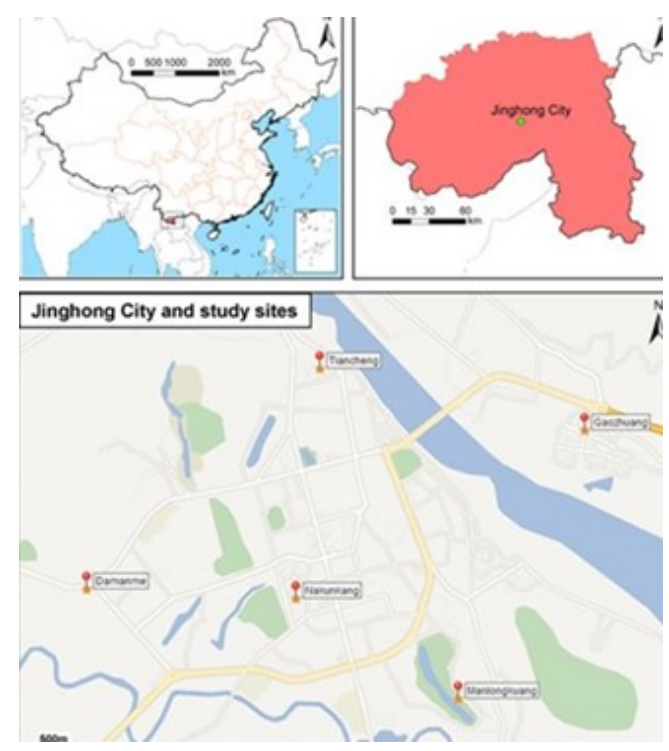

Figure 1: The location of Jinghong city and study sites.

Mosquito survey: A door to door cross-sectional entomological survey was carried out in houses and peridomestic areas to detect Aedes larval breeding. The larval collections were made in each locality by using dipping and pipetting methods, to find out Aedes breeding in all water filled containers present in and around the houses and their premises in studied areas. The data on larval collections were recorded in the pre-designed and pre-tested survey forms. Each water container with larvae was moved to a bottle marked with a label consistent with the record.

From February to March, and from November to December, we surveyed at least once a month. From April to October, we surveyed 3 times a month, 7-15 days intervals between two surveyed works. No less than 50 houses and peridomestic sites were surveyed in each site each time.

Laboratory evaluation: Every bottle with collected larvae labeled was taken to the laboratory. We randomly dipped 5-10 larvae on a slide for species identify by the taxonomic key [2,7]. Then Aedes species was recorded in the pre-designed survey forms. From February to May, we recorded the total Aedes number (for Ae. aegypti and Ae. albopictus). From June to December, we identified species for Ae. aegypti and Ae. albopictus separately and recorded.

Statistical analysis: The data on larval collections were analysed and different indices like container index (CI) [8] and breteau index (BI) [4] were calculated. CI is the composition of positive containers with larvae of Aedes by all water containers. BI is the larva index of Aedes density, which means the number of positive containers when checking one hundred houses. Container preference of Aedes larval breeding was also assessed by calculation of breeding preference ratio. The differences in breeding found indoor and outdoor were tested for significance by different species. The distribution of both species in different clusters was tested by SPSS. The seasonal variation of the water quantity in 100 households (WH), BI and CI were analyzed. Monthly changes were described and analyzed with influencing factors.

\section{Results}

During our survey in Jinghong city in 2014, we made a total of 6609 houses and peridomestic survey records in the 5 clusters. In total 12391 water holding containers were identified, of which 1161 (9.37\%) had Aedes mosquito breeding, the CI was 9.37 and the BI was 17.57 in annual average value.

\begin{tabular}{|l|l|l|l|l|l|l|l|l|}
\hline Site & BI & CI & Houses & Containers & Containers+ (\%) & Alb+ (\%) & Aeg+ (\%) & Both+ (\%) \\
\hline D & 13.67 & 10 & 1266 & 1697 & $173(10.19)$ & $75(43.35)$ & $79(45.66)$ & $9(5.20)$ \\
\hline G & 25.89 & 13 & 1240 & 2388 & $321(13.44)$ & $24(7.48)$ & $215(66.98)$ & $14(4.36)$ \\
\hline M & 23.81 & 15 & 1285 & 2096 & $306(14.60)$ & $173(56.54)$ & $94(30.72)$ & $20(6.54)$ \\
\hline N & 5.52 & 5 & 1341 & 1351 & $74(5.48)$ & $20(27.03)$ & $41(55.41)$ & $7(9.46)$ \\
\hline T & 19.43 & 6 & 1477 & 4859 & $287(5.91)$ & $16(5.57)$ & $218(75.96)$ & $13(4.53)$ \\
\hline Total & 17.57 & 9 & 6609 & 12391 & $1161(9.37)$ & $308(26.53)$ & $647(55.73)$ & $63(5.43)$ \\
\hline
\end{tabular}

Note: D: Damanme; G: Gaozhuang; M: Manlongkuang; N: Nakunkang; T: Tiancheng. Total: total number; Containers: number of water containers summarized by different communities. Containers+ (\%): number of positive containers and the percentage of total containers; Alb+: Number of positive containers with Ae. albopictus; Aeg+: Number of positive containers with Ae. aegypti; Both+: Number of containers with both Ae. albopictus and Ae. Aegypti.

Table 1: Positive water containers and indices by different communities.

From June to December, there were 647 positive containers with $A$. aegypti include 480 (74.19\%) indoor and 167 (25.81\%) outdoor. Tires took up to $60.48 \%$ in the outdoor positive containers. If we remove the
101 tires outdoor, there were 66 outdoor containers that took up only $12.09 \%$. 
Citation: Guo YH, Lai SH, Huange Q, Rena DS, et al. (2016) Coexistence of Aedes aegypti and Aedes albopictus in Jinghong City, Yunnan Province: A Survey of Aedes aegypti Invasion. J Trop Dis 4: 227. doi:10.4172/2329-891X.1000227

Page 3 of 6

There were 308 positive containers by Ae. albopictus including 140 (45.45\%) indoor and $168(54.55 \%)$ outdoor with 38 (22.62\%) distributed in tires. Out of these, 63 had mixture of the both species. Two mosquito species composition ratio was about 2 to 1 by $A e$. aegypti and Ae. albopictus, and the mixed container took up 5.43\%.

The average number of water containers per house in different communities during the study was different; Tiancheng had the highest number of water containers per house (3.3), and in turns by Gaozhuang (1.9), Manlongkuang (1.6), Damanme (1.3), and Nakunkang (1.0). Positive water containers composition varied from $5.48 \%$ to $14.60 \%$, Manlongkuang (14.60\%), Gaozhuang (13.44\%), Damanme $(10.19 \%)$ had relatively high positive composition, while Tiancheng (5.91\%) and Nakunkang (5.48\%) showed with a relatively less positive composition.
The average $\mathrm{BI}$ and $\mathrm{CI}$ by different localities were provided in Table 1. Both BI $(\mathrm{F}=4.83, \mathrm{P}=0.001)$ and $\mathrm{CI}(\mathrm{F}=4.20, \mathrm{P}=0.003)$ in different communities showed statistically significant differences. The higher value existed in Gaozhuang and Manlongkuang and DamanmeTiancheng and Nakunkang with relatively lower value.

The composition of both species showed difference by different communities $(\chi 2=271.61, \mathrm{P}<0.001)$. Tiancheng $(75.96 \%, 218 / 287)$, Gaozhuang $(66.98 \%, 215 / 321)$, Nakunkang $(55.41 \%, 41 / 74)$ had a high composition of Ae. aegypti, whereas Manglongkuang had higher proportion composition of Ae. Albopictus (56.54\%, 173/306), while the proportions of the two species were almost equivalent in Damanme.

\begin{tabular}{|l|l|l|l|l|l|l|l|l|l|}
\hline Container & Total & F & P & PA & O & J & W & T & B \\
\hline Total & 12391 & 5067 & 2418 & 1925 & 1056 & 746 & 638 & 494 & 30 \\
\hline Positive & $1161(9.37 \%)$ & $330(6.51 \%)$ & $269(11.12 \%)$ & $235(12.21 \%)$ & $\begin{array}{l}64 \\
(6.06 \%)\end{array}$ & $\begin{array}{l}29 \\
(3.89 \%)\end{array}$ & $\begin{array}{l}87 \\
(13.64 \%)\end{array}$ & $139(28.14 \%)$ & $6(20.00 \%)$ \\
\hline
\end{tabular}

Note: P: pots in houses; F: flower pots; T: tires; B: bamboo; J: jars; PA: pail; W: Waste bottle; O: other containers, such as pedal of a tricycle, waste package of snack, and so on.

Table 2: Number of water bodies and positive (composition) by different types.

For total number of water containers distributed in the five communities, the highest positive composition was tires, which amounted to $28.14 \%$. The second was bamboo cuts ( $20.00 \%$ ), followed by waste bottles (13.64\%), the pails (12.21\%), the pots for household use $(11.12 \%)$, the flower pots $(6.51 \%)$, and lastly the jars $(3.89 \%)$.

From the total number of view, the most productive breeding sites for dengue vectors were the most abundant ones (Table 2). There was the highest number of flower pots (5067) with the highest positive number (330), followed by pots in houses (269/2418), Pail (235/1925), waste bottles (87/638), and Bamboo (6/30). However, jars in houses showed positive of $29 / 746$, the larger number water containers but with less positive. Tires showed high positive of 139/494 indicating preferred breeding sites for Aedes.

\begin{tabular}{|l|l|l|l|l|l|l|l|l|l|l|l|}
\hline & Feb & Mar & Apr & May & Jun & Jul & Aug & Sept & Oct & Nov & Dec \\
\hline WH & 131 & 114 & 125 & 177 & 232 & 220 & 215 & 183 & 218 & 218 & 202 \\
\hline BI & 4 & 1 & 6 & 11 & 21 & 27 & 45 & 21 & 9 & 19 & 15 \\
\hline Cl & 3 & 1 & 5 & 6 & 9 & 12 & 21 & 12 & 4 & 9 & 8 \\
\hline
\end{tabular}

Note: WH: water quantity in 100 households; Cl: composition of positive containers with larva of Aedes by all water containers; Bl: number of positive containers in one hundred houses.

Table 3: Monthly water containers number and index of Aedes.

The monthly average $\mathrm{BI}$ and $\mathrm{CI}$ and the temporal variance by monthly average number were provided in Table 3 . The smallest index happened in March. Number of water containers increased suddenly from April, reaching the highest in June, and maintained a high value until November. The number of positive containers showed a smooth increase trend from March, reaching the highest in August, and kept high value until December. Indices of BI and CI showed the similar trends.

\section{Discussion}

The larval survey is the most widely used method for entomological surveillance, for practical reasons when compared to egg, pupal and adult surveys [9], especially to the vector Aedes for dengue,
Chikungunya, and Zika viruses [10]. We designed an annual dengue vector larval survey in Jinghong city in 2014 and got species identification from June to December. Generally, Ae. egypti was found at a higher frequency than Ae. albopictus in these areas. Lu [2] reviewed almost all the published papers up to 1993 for thorough mosquito investigation throughout China during 1980s, and there was no record for the distribution of Ae. aegypti in Yunnan province, he also described the distribution of Ae aegypti in China. For this reason, some reports on global Ae. aegypti distribution did not include its distribution in China [5,6]. The distribution of Ae. aegypti in Xishuangbanna was firstly found in 2013 during dengue epidemic there [1]. From the surveillance results from June to December, 2014, there were 647 positive containers for Ae. aegypti while 308 for Ae. 
albopictus. Out of these, there were 63 water containers with both species. Aedes aegypti amounted to $67.75 \%$ in the total number of positive Aedes larval containers. The results showed that Ae. aegypti has invaded and occupied a considerable composition since the vague time of its first invasion to Jinghong city, and coexists with Ae. albopictus sharing the preferred breeding sites. The results were in agreement with the studies [5]. Our results will fill in the gaps in the Ae. aegypti distribution in Xishuangbanna, Yunnan province.

There were 63 water containers with both Ae. albopictus and Ae. Aegypti. Mixed breeding indicates that more than one mosquito species can oviposit in a single container and both species share the same breeding sites in nature (at least to some extent), there must be competitive between both species. The competition between Ae. aegypti and Ae. albopictus showed different alternatives in different places. Several authors have implied that through competition or some other factor the invasion of South-East Asia by Ae. Aegypti had an effect on the populations of Ae. Albopictus. Calcutta was described with "stale equilibrium" of both species and deduced that conclusion by [11], and it was [12] found there only $5 \%$ of the two species were Ae. albopictus. Studies [13] suggested that Ae. aegypti is favored by rapid and extensive urbanization and by the higher fecundity and shorter life cycle of the species in Singapore city. It is also possible that Ae. albopictus may have an adverse effect on Ae. aegypti. Studies [14] presented that although Ae. aegypti had the advantage of arriving 1st than Ae. albopictus, Ae. aegypti did not remain the predominant mosquito in Honolulu. Recent studies [15] reported that Ae. aegypti has decreased from high prevalence in 1907 to relict populations in remote valleys today in Mayotte. It was [16] reported that in certain human dwellings/habitats, Ae. albopictus were ovipositing inside the premises and was predominant in population compared to Ae. Aegypti, these patterns of decline in Ae. aegypti might be typical after the introduction of Ae. albopictus.

The relationship between Ae. aegypti and Ae. albopictus is therefore of more than casual interest. We appeared to be observing in Jinghong the type of competitive displacement of both species. Aedes aegypti had occupied the most habitats than Ae. albopictus, and also there were the overlapping breeding sites taking up to $6.60 \%$. Aedes aegypti is the superior competitor in domestic premises since its invasion when sharing the same breeding containers. From zero to the present proportion of Ae. aegypti in Jinghong city, we further deduced that $A e$. aegypti would expand its distribution quickly. We did not know the mechanism for Ae. aegypti to spread widely in recent years, but it has invaded and colonized in Jinghong city and further spread here might be disputable. In our studies, two mosquito species were found in all types of water samples, indicating a common breeding ground for both species. Thus the principle of competitive exclusion appears to be in operation in the extreme parts of the shared habitat. The possibility cannot be excluded that the invasion of Ae. aegypti in Jinghong city might lead to a decrease or eradication of Ae. albopictus in houses and dwellings. As for how interspecific competition happened in the future, it could only be verified by the further monitoring results, and the results would be decided by ecological factors and the interventions. We would agree [11] that competition with Ae. albopictus would not prevent Ae. aegypti from becoming established in an urban environment, and also that the dependence of this species on man discourages it from invading the habitats of Ae. albopictus. Not only are the relative prevalence of these species in a given area of importance in the epidemiology of viral diseases, but they may represent another example illustrative of a basic biological principle.
There are many factors which make up the ecological niche, and in the case of these two mosquitoes the essential elements are shelter and food for the adults and breeding-habitats for the larvae. The changes in the relative proportions of the two mosquito populations must have been caused in part by the differential effect of control measures.

We explored whether the most productive breeding sites for dengue vectors were the most abundant breeding sites because it is more practical to target to eliminate the most common type of breeding sites in Jinghong city. As in our conclusion, that all types of water containers can become a breeding ground for vector Aedes. Number of positive Aedes water containers showed in terms with the number of water containers, except tires with high and jars with less positive proportion. Aedes mosquito showed its preference with tires, the positive composition took up to $28 \%$. The results are consistent with that of research papers on breeding sites [17-20]. The lowest positive jars in house in our results might have relevant with the vector control work in 2013. We emphasized the importance of cleaning jars generally used for pickle in these areas during dengue vector control in 2013. We recommended weekly cleaning for household plants, covering the pickle jar with a plastic bag, and avoiding long-term household water storage containers that serve as larval habitat for dengue mosquitoes. Treatment of pickle jars might affect relative smaller positive rate in the results.

For dengue, a disease without drugs or vaccines, the main approach used in prevention and control is reducing the population of Aedes mosquito [4,21]. During the dengue epidemic in China, we had an intervention strategy to suppress the dengue mosquito vectors $[4,22]$. The effective vector control measures are critical to achieve and sustain the reduction of morbidity attributable to dengue. And integrated vector management (IVM) is the key measure for dengue control [23]. We hypothesized that most vectors reproduced in all types of water containers breeding sites in houses and peridomestic areas, dengue vector control strategies comprised adult-killed quickly and breeding elimination of all types of water containers both indoors and outdoors [4]. In our results, dengue vectors (Ae. aegypti and Ae. albopictus) could breed in all kinds of water containers surveyed in 2014. Aedes aegypti were more favored indoors, while Ae. albopictus like both. In common, it is harder to eliminate the vectors indoors than outdoors. During our previous work for dengue vector control, the staffs engaged in the works would rather do more work outdoors than the controlling work indoors due to the difficult to enter a house. Even, some specialist takes the ideas that a complete eliminating work outdoors could instead of works indoors. The results gotten from our study, the indoors distribution took up to $74.19 \%$ for Ae. aegypti, and $45.45 \%$ for Ae. albopictus separately. These further confirmed the importance of the indoors controlling work for dengue vector, especially for the main vector Ae. aegypti, even for the second vector Ae. albopictus, dengue vector control indoors is the same important as outdoors. We insist on the vector control to eliminate all types of small water bodies indoors and its peridomestic areas. Aedes vector could breed in all kinds of water containers. If left some untreated, it will produce sufficient vector to make dengue fever epidemic.

Temporal distribution of Aedes might be consistent with climate change in Jinghong city. Jinghong city has a tropical monsoon climate; it is located in the south of the Tropic in China, with high temperatures throughout the year. There are two seasons (rainy and dry seasons) a year. The rainy season begins in March, gets a stable from May to October to late, and then decreased in November. The dry season keeps from November to March. The rainy season precipitation 
accounted for $80 \%$ of annual precipitation. The temporal variance might consistent with the raining. Number of water containers increased suddenly from March, and reach to the highest in June, and maintained a high value until October, then suddenly dropped in November. The number of positive containers showed a smooth curve, rose since February, highest in August, maintained until November, and then decreased. Indices of BI and CI showed with similar trends. Our results showed a decrease in October. This might be affected by the field test for adult-killing chemical space spray work carried there in Jinghong city from mid - September to early-October, 2014 (internal information). On the basis of the vector density threshold for dengue transmission risk $[7,24]$, and by terms of BI got from our surveillance in Jinghong city, there almost no risk of dengue transmission in February and March; April, May, and December has the lower risk dengue prevalence; while from June to November with a high risk of transmission. Of course, the average number of water containers per house in different communities during the study is different, we must emphasize that disease epidemic has closely related with the local population and vector density once case imputed.

There are some limitations in this study. We abandoned the other mosquito species collected belonging to Culex despite its small number, which do not have relations with dengue. We have not investigated the sewer as Aedes habitats verified by [25]. We could not distinguish the two vectors morphologically before May, 2014. We have no evidence suggesting that differential preference sites for both adult mosquitoes in our study, which could be getting in a way of examining trap preferences, would be to compare relative densities of each species in a given type of trap. This would help us to control the adult during the epidemic time. This will further study in the future.

In conclusion, larval surveillance could found the most productive breeding sites for dengue vectors Ae. aegypti and Ae. albopictus. Both vectors could breed in all kinds of small water containers in houses and peridomestic areas. Both prefer tires, and the vector abundance was consistent with the number of water containers. The results of competition between the two vector species will be decided by environmental and control measures. Seasonally, the rainy season in Xishuangbanna will have a higher risk of dengue fever. Of course, we should give a detailed understanding of characters by population and vector density once disease epidemic there. Vector control management should focus on all types of water containers indoor and outdoor. This is relevant for both the national dengue prevention and control program and the special program for research and training in tropical diseases of the WHO.

\section{Ethical Approval}

Ethical approval for this study was obtained from the Ethical Committee of China CDC (No.201214).

\section{Authors' Contributions}

GYH worked as the technical guidance, designed and participated field investigating work at the beginning of the field work, and carried out statistical analysis and wrote the paper. SJL finalized the analysis, wrote the manuscript, and interpreted the findings. HQ and ZJH took charge of the field investigating work and organized the group investigating by the same method. RDS provides a good proposal for the manuscript and participated the field work. LQY organized the work. ZHQ helped to carry out statistical analysis and gave his comments on the paper. All authors approved the final version of the manuscript.

\section{Conflict of Interest}

The authors declare that they have no competing interests.

\section{Acknowledgements}

The study was supported by the National Basic Research Program of China (973 Program) (Grant No. 2012CB955504); the National Natural Science Foundation of China (NSFC) (Grant No. 81273139); the Ministry of Science and Technology of China (2016ZX10004222-009). The funder had no role in study design, data collection and analysis, decision to publish, or preparation of the manuscript, and the content is solely the responsibility of the authors and does not necessarily represent the official views of it. We are debt to Dr Yu-Juan Yue from Department of Vector Biology and Control, ICDC, China CDC, for her help in data analysis. The authors would like to thank Jing-li Liu, Gong-hui Jiang at Department of Vector Biology and Control, ICDC, China CDC, and all the staffs' participant the field investigating for Aedes larvae distribution work in Jinghong city who gave their help during the work.

\section{References}

1. Zhang FC, Zhao H, Li LH, Jiang T, Hong WX, et al. (2014) Severe dengue outbreak in Yunnan, China. International journal of infectious diseases 27: 4-6.

2. Lu BL (1997) Fauna Sinica Insecta. Vol. 8, Diptera: Culicidae. Science Press, Beijing, China.

3. Wu F, Liu Q, Lu L, Wang J, Song X, et al. (2011) Distribution of Aedes albopictus (Diptera: Culicidae) in northwestern China. Vector borne and zoonotic diseases 11: 1181-1186.

4. Guo YH, Lai SJ, Liu XB, Li GC, Yu HJ, et al. (2016) Governmental supervision and rapid detection on dengue vectors: An important role for dengue control in China. Acta tropica 156: 17-21.

5. Campbell LP, Luther C, Moo LD, Ramsey JM, Danis LR, et al. (2015) Climate change influences on global distributions of dengue and chikungunya virus vectors. Philosophical transactions of the Royal Society B, Biological sciences, London.

6. Kraemer MU, Sinka ME, Duda KA, Mylne AQ, Shearer FM, et al. (2015) The global distribution of the arbovirus vectors Aedes aegypti and Ae. albopictus. eLife 4: 08347.

7. Xiao DLLH, Li DX (2008) Handbook for the prevention and treatment of dengue fever. Disease Control and Prevention Bureau, Chinese Ministry of Health.

8. Zhao TYDY, Liu MD (2009) Servillance methods for vector densitymosquito.

9. http://www.searo.who.int/entity/vector_borne_tropical_diseases/ documents/SEAROTPS60/en/

10. Yalwala S, Clark J, Oullo D, Ngonga D, Abuom D, et al. (2015) Comparative efficacy of existing surveillance tools for Aedes aegypti in Western Kenya. Journal of vector ecology 40: 301-307.

11. Gilotra SK, Rozeboom LE, Bhattacharya NC (1967) Observations on possible competitive displacement between populations of Aedes aegypti Linnaeus and Aedes albopictus Skuse in Calcutta. Bulletin of the World Health Organization 37: 437-446.

12. Tandon N, Ray S (2000) Breeding habitats and larval indices of Aedes aegypti and Ae. albopictus in the residential areas of Calcutta City. The Journal of Communicable Diseases 32: 180-184.

13. Chan KL, Chan YC, Ho BC (1971) Aedes aegypti (L.) and Aedes albopictus (Skuse) in Singapore City. 4. Competition between species. Bulletin of the World Health Organization 44: 643-649. 
Citation: Guo YH, Lai SH, Huange Q, Rena DS, et al. (2016) Coexistence of Aedes aegypti and Aedes albopictus in Jinghong City, Yunnan Province: A Survey of Aedes aegypti Invasion. J Trop Dis 4: 227. doi:10.4172/2329-891X.1000227

Page 6 of 6

14. Winchester JC, Kapan DD (2013) History of Aedes mosquitoes in Hawaii. Journal of the American Mosquito Control Association 29: 154-163.

15. Bagny L, Delatte H, Elissa N, Quilici S, Fontenille D, et al. (2009) Aedes (Diptera: Culicidae) vectors of arboviruses in Mayotte (Indian Ocean): distribution area and larval habitats. J Med Entomol 46: 198-207.

16. Misni N, Sulaiman S, Othman H, Omar B (2009) Repellency of essential oil of Piper aduncum against Aedes albopictus in the laboratory. J Am Mosq Control Assoc 25: 442-447.

17. Ferdousi F, Yoshimatsu S, Ma E, Sohel N, Wagatsuma Y, et al. (2015) Identification of essential containers for aedes larval breeding to control dengue in Dhaka, Bangladesh. Tropical Medicine and Health 43: 253-264.

18. Getachew D, Tekie H, Gebre MT, Balkew M, Mesfin A (2015) Breeding sites of Aedes aegypti: potential dengue vectors in Dire Dawa, East Ethiopia. Interdiscip Perspect Infect Dis 2015: 706276.

19. Hertz JT, Lyaruu LJ, Ooi EE, Mosha FW, Crump JA (2016) Distribution of Aedes mosquitoes in the Kilimanjaro Region of northern Tanzania. Pathog Glob Health 110: 108-112.
20. Bartlett HK, Unlu I, Obenauer P, Hughes T, Healy S, et al. (2012) Larval mosquito habitat utilization and community dynamics of Aedes albopictus and Aedes japonicus (Diptera: Culicidae). J Med Entomol 49: 813-824.

21. Andersson N, Nava AE, Arostegui J, Morales PA, Suazo LH, et al. (2015) Evidence based community mobilization for dengue prevention in Nicaragua and Mexico (Camino Verde, the Green Way): cluster randomized controlled trial. Bmj 351: 3267

22. Wang T, Wang M, Shu B, Chen XQ, Luo L, et al. (2015) Evaluation of inapparent dengue infections during an outbreak in Southern China. PLoS Negl Trop Dis 9: e0003677.

23. http://www.who.int/denguecontrol/9789241504034/en/

24. https://www.cdc.gov/dengue/

25. Cao HG, Fan J, Jin S, Zhou Y, Leng P, et al. (2016) Mosquito breeding and counter measures in underground garages in Huangpu district, Shanghai. Chin J Vector Biol \& Control 27: 155-159. 